\title{
DEWEY Y EL MITO DE LO DADO
}

\section{DEWEY AND THE MYTH OF THE GIVEN*}

\author{
Daniel KALPOKAS** \\ CONICET-UNC-UBA
}

\begin{abstract}
RESUMEN: El presente artículo reconstruye la crítica de Dewey al empirismo clásico como una crítica a lo que Sellars llama «el Mito de lo Dado». En primer lugar, se caracteriza sucintamente el Mito de lo Dado. En segundo lugar, se expone la concepción experimental de la experiencia de Dewey y sus principales críticas a la concepción de la experiencia sustentada por el empirismo tradicional. En tercer lugar, se muestra cómo tales críticas pueden entenderse como objeciones al Mito criticado por Sellars. Finalmente, se destacan algunas diferencias entre las posiciones de Sellars y Dewey que, en cierto modo, hacen preferible la propuesta de este último.
\end{abstract}

Palabras Clave: Dewey, Sellars, Mito de lo Dado, concepción experimental de la experiencia.

ABSTRACT: This paper reconstructs Dewey's criticism to classic empiricism as a criticism to what Sellars calls «the Myth of the Given». Firstly, the Myth of the Given is characterized. Secondly, Dewey's experimental conception of experience and his main objections to traditional empiricism are described. Thirdly, it is showed how those objections can

* El presente artículo ha sido escrito en el marco de los siguientes proyectos de investigación: «Experiencia y conceptos: un enfoque pragmatista», subsidiado por la Secretaría de Ciencia y Tecnología de la Universidad Nacional de Córdoba (período 2010-2012), y «Autoconocimiento y auto-referencia", subsidiado por el Consejo Nacional de Investigaciones Científicas y Técnicas (período 2009-2011).

** e-mail: dkalpokas@gmail.com 
be understood as objections to the Myth criticized by Sellars. Finally, some differences between Sellars and Dewey are stressed. In certain way, those differences make preferable Dewey's proposal.

KeYwORDS: Dewey, Sellars, Myth of the Given, experimental conception of experience.

\section{Introducción}

En «Empiricism and the Philosophy of Mind» Sellars presenta su ataque a lo que denomina «el Mito de lo Dado». Dicho ataque (que ha tenido una enorme influencia en la filosofía norteamericana del siglo XX) se apoya en algunas intuiciones características de los filósofos que pertenecieron al giro lingüístico. Unas décadas antes, sin embargo, Dewey había presentado - en diversas partes de su obra- su propia crítica del empirismo clásico. Empero, en vez de apoyarse en el análisis del lenguaje, Dewey desarrolló su crítica a partir de lo que él consideraba que era una concepción empírica de la experiencia. Aunque se desarrollen por carriles diferentes, pienso que las críticas de Sellars y Dewey pueden entenderse como apuntando al mismo blanco. Así pues, en este trabajo pretendo reconstruir la crítica de Dewey al empirismo clásico como una crítica al llamado «Mito de lo Dado». Espero que indirectamente dicha reconstrucción ponga de manifiesto los aciertos y la actualidad de la filosofía de Dewey en los debates contemporáneos sobre la percepción en filosofía de la mente y en epistemología ${ }^{1}$. En la sección (2) reconstruyo sucintamente el Mito de lo Dado tal como aparece en «Empiricism and the Philosophy of Mind». En la sección (3) expongo la concepción experimental de la experiencia y las principales críticas de Dewey a la concepción de la experiencia sustentada por el empirismo tradicional. En la

1 Rorty, que ha hecho mucho por mostrar la actualidad de la obra de Dewey, considera, sin embargo, que la noción deweyana de experiencia es una rémora del pasado que hay que dejar atrás. (Cf. Rorty, R., "Overcoming the Tradition: Heidegger and Dewey» y "Dewey's Metaphysics», en Rorty, R., Consequences of Pragmatism, Minneapolis, University of Minnesota Press, 1982; $\mathrm{y}$ «Dewey Between Hegel and Darwin», en Rorty, R., Truth and Progress, Cambridge, Cambridge University Press, 1998). Como dice Bernstein en su último libro, el pragmatismo de Rorty es un "pragmatismo sin experiencia». Cf. Bernstein, R., The Pragmatic Turn, Cambridge, Polity Press, 2010, cap. 6. En contraposición, creo que la noción deweyana de «experiencia» es todavía una noción útil para muchos debates contemporáneos en filosofía de la mente y en epistemología. 
sección (4) muestro cómo tales críticas pueden entenderse como objeciones al Mito criticado por Sellars. Finalmente, la sección (5) sintetiza los principales resultados obtenidos en las secciones precedentes.

\section{Sellars y el Mito de lo Dado}

A pesar de la influencia que ha tenido la crítica de Sellars al Mito de lo Dado², no resulta sencillo identificar el blanco de sus argumentos ${ }^{3}$. Sellars aclara que el marco de lo dado abarca tanto contenidos sensoriales, como objetos materiales, universales, proposiciones y primeros principios, aunque su ataque se concentra en la teoría de los datos sensoriales.

Según Sellars, la categoría epistemológica de «lo dado» ha sido introducida para explicar la idea de que el conocimiento empírico se fundamenta, en última instancia, en cierto tipo de conocimiento no inferencial. Su crítica se dirige, no a la noción misma de "conocimiento no inferencial» (pues Sellars sostiene que los reportes observacionales como «Esto es rojo» constituyen ejemplos legítimos de conocimiento no inferencial), sino a la idea de conocimiento no inferencial

2 Piénsese, por ejemplo, en el uso que hace Rorty de la filosofía de Sellars en el cap. 4 de Philosophy and the Mirror of Nature, Princeton, Princeton University Press, 1979; en el inferencialismo semántico de Brandom en Making it Explicit, Cambridge, Harvard University Press, 1994; y en las lecturas que McDowell ha hecho de la obra de Sellars en Having the World in View, Cambridge, Harvard University Press, 2009. Para una crítica de los argumentos que Sellars esgrime en contra del mito de lo dado, Cf., por ejemplo, Alston, W., «What's Wrong with Immediate Knowledge?», Synthese 55, 1983, y Bonevac, D., "Sellars vs the Given", Philosophy and Phenomenological Research, vol. LXIV, n. ${ }^{\circ} 1,2002$.

3 Bonevac distingue cinco tesis involucradas en el llamado Mito de lo Dado: 1) la tesis de que los episodios internos no presuponen capacidades conceptuales adquiridas; 2 ) la tesis de que algunos episodios internos constituyen conocimiento no inferencial; 3) la tesis de que la percepción sensible [sensing] es una condición necesaria para el aprehender [grasping]; 4) la tesis de que la aprehensión es una condición necesaria de todo otro conocimiento empírico; y 5) la tesis de que la percepción sensible [sensing] juega un rol en la justificación de las aprehensiones. Cf. Bonevac, "Sellars vs the Given», Philosophy and Phenomenological Research, vol. LXIV, n. ${ }^{\circ}$ 1, 2002, p. 2 y 3. Sea correcta o no la reconstrucción que hace este autor de la posición de Sellars, para mis propósitos basta con exponer lo que — según mi entender y el de autores que han seguido a Sellars en este punto- constituye el núcleo del Mito de lo Dado. Para una comparación entre el ataque de Sellars al Mito de lo Dado y el ataque de Peirce al cartesianismo, Cfr. Bernstein, R., op. cit. «Prólogo» y cap. 1 «Charles S. Peirce's Critique of Cartesianism». 
dado en la experiencia que es independiente de las capacidades conceptuales adquiridas y que, no obstante, podría servir para justificar las creencias que versan sobre el mundo. El Mito de lo Dado aparece, pues, como la idea según la cual cierta clase de hechos no epistémicos acerca de los sujetos cognoscentes podrían implicar hechos epistémicos acerca de ellos ${ }^{4}$. Este es un error que, según Sellars, es de la misma índole que la llamada «falacia naturalista» en ética ${ }^{5}$. Los mencionados hechos no epistémicos («naturales» los llama también Sellars) ${ }^{6}$ consisten, en general, en la aparición inmediata de objetos ante la mente, objetos con respecto a los cuales la mente no puede sino tener una conciencia directa. Así, por ejemplo, de acuerdo a la teoría de los datos sensoriales (que es una de las teorías filosóficas que favorece el surgimiento del Mito), la aprehensión de los contenidos mentales se lleva a cabo sin mediación del lenguaje. Desde esta concepción, la mente es el dominio de lo que es conocido inmediatamente (entendiendo por tal conocimiento, no meramente el conocimiento no inferencial, sino aquel que supone una aprehensión de lo que le es dado a la mente que excluye la posibilidad del error y de la ignorancia). De este modo, según Sellars, los teóricos de los datos sensoriales tienden a hacer equivalentes el percibir contenidos sensoriales (algo que compartimos con las criaturas pre-lingüísticas) y el ser conciente (algo que es una instancia de conocimiento y que, por ende, presupone la capacidad de justificar aserciones).

La caracterización que da Sellars de la noción de «dato sensorial» puede utilizarse para caracterizar también el núcleo esencial del Mito de lo Dado. Según sostiene Sellars, el concepto de «dato sensorial» resulta de la articulación de las siguientes dos ideas:

ii) la idea de que hay ciertos episodios internos (i.e. sensaciones de rojo) que pueden tener lugar en la mente humana sin necesidad de conceptos, y sin

${ }^{4}$ En «Sellars on Perceptual Experience», McDowell interpreta que «epistémico» equivale a algo así como «que involucra conceptos». Así, entiende que la idea principal de «Empiricism and the Philosophy of Mind» es que "no debemos suponer que podemos comprender los estados o episodios epistémicos en términos de la actualización de meras capacidades naturales — capacidades que sus sujetos tienen desde su nacimiento-, o adquirido en el curso de la mera maduración animal». McDowell, J., Having the World in View, p. 5.

5 Sellars, W., Empiricism and the Philosophy of Mind, Cambridge, Harvard University Press, 1997, \# 5, p. 19.

${ }^{6}$ Cf. Sellars, W., op. cit., \# 17, p. 42. 
los cuales sería imposible tener experiencia alguna (i.e. de que la cara de un objeto físico es roja y triangular); y

ii) la idea de que hay ciertos episodios internos que constituyen conocimiento no inferencial, y que son condiciones necesarias del conocimiento empírico pues proveen la evidencia (última) de toda proposición empírica ${ }^{7}$.

Tenemos aquí todos los elementos que hacen al llamado "Mito de lo Dado»: a) la idea de ciertos episodios internos, necesarios para tener experiencias, que no requieren conceptos; b) la tesis de que tales episodios constituyen un caso de conocimiento no inferencial; y c) la tesis fundacionista según la cual tales episodios proveen los fundamentos últimos de todo nuestro conocimiento empírico. Sellars acepta que ciertos episodios internos como una sensación de rojo tienen lugar en nosotros sin necesidad de conceptos, aunque subraya que tales episodios no constituyen conocimiento alguno (no son hechos epistémicos). También reconoce que esos episodios desempeñan un papel en la experiencia, a saber, un papel causal. Asimismo, está dispuesto a aceptar que hay ciertos episodios internos que constituyen conocimiento no inferencial, siempre y cuando se reconozca que tal conocimiento presupone el uso del lenguaje. Lo que Sellars rechaza (porque parece constituir el núcleo del Mito) es que exista un tipo de conocimiento no inferencial que no presuponga el uso de concepto alguno (y, por tanto, que no presuponga el uso del lenguaje), y que constituya la base de justificación última de todo el conocimiento empírico. En efecto, una de las formas que adopta el Mito consiste, según Sellars, en la idea de que hay una estructura formada por cuestiones de hecho singulares tal que (i) puedan conocerse no inferencialmente tales cuestiones de hecho sin presuponer otro tipo de conocimiento (ya sea sobre cuestiones fácticas singulares o sobre verdades generales); y (ii) tal conocimiento no inferencial constituya la instancia última de apelación para todas las cuestiones fácticas. Frente a esto, Sellars muestra, no sólo que la idea de conocimiento no inferencial no conceptual es incoherente, sino, además, que la tesis fundacionista que sostiene que habría un tipo de conocimiento no inferencial que no presupone conocimiento alguno de otras cuestiones fácticas, es insostenible. La pretendida autoridad del conocimiento «último» descansa, en verdad, en el conocimiento de otras cuestiones de hecho (singulares y generales) ${ }^{8}$.

\footnotetext{
7 Cf. Sellars, W., op. cit., \# 7, p. 21 y 22.

${ }^{8}$ Cf. Sellars, W., op. cit., \# 38 y ss.
} 
Frente a la concepción del conocimiento que sustenta al Mito de lo Dado, Sellars afirma:

El punto esencial es que, al caracterizar un episodio o un estado como de conocer, no estamos dando una descripción empírica de ese episodio o estado; estamos ubicándolo en el espacio lógico de las razones, del justificar y del ser capaz de justificar lo que uno dice?.

No hay, pues, para Sellars, conocimiento que no involucre la habilidad para manejar conceptos; $y$, puesto que la posesión de un concepto es identificada por Sellars con el dominio del uso de una palabra, no puede haber, por tanto, conocimiento sin uso de lenguaje. El lenguaje constituye un prerrequisito de la experiencia consciente. Como ha dicho Rorty:

No hay una cosa tal como la creencia justificada que no sea proposicional, y no hay justificación que no sea una relación entre proposiciones. Siempre será un error hablar de nuestro contacto [acquaintence] con la rojez o con una instanciación de la rojez como si fuera el «fundamento» (en oposición a ser una condición causal) de nuestro conocimiento de que «esto es un objeto rojo» o de que «el rojo es un color» ${ }^{10}$.

No importa aquí reconstruir los complejos y variados argumentos de Sellars en contra del Mito de lo Dado ${ }^{11}$. El propósito del presente vistazo a «Empiricism and the Philosophy of Mind» ha sido meramente el de tratar de caracterizar el blanco de las ulteriores críticas que se desarrollan en dicho artículo e indicar cuál es la concepción del conocimiento que Sellars contrapone a la sustentada por el Mito. Veamos, pues, ahora, cuál es la crítica de Dewey al empirismo tradicional y cómo podría entenderse como una crítica al Mito cuestionado por Sellars.

\footnotetext{
9 Sellars, W., op. cit., \# 36, p. 76.

${ }_{10}$ Rorty, R., Philosophy and the Mirror of Nature, Princeton, Princeton University Press, 1979, p. 183.

11 En oposición a lo afirmado por Brandom en su «Study Guide» (en Sellars, W., Empiricism and the Philosophy of Mind, Cambridge, Harvard University Press, 1997) sobre «Empiricism and the Philosophy of Mind», en donde Brandom afirma que en ese artículo la posición de Sellars con respecto al empirismo no es clara, McDowell afirma, en «Why Is Sellars's Essay Called "Empiricism and the Philosophy of Mind"?», que, en verdad, Sellars intenta mostrarnos cómo ser «buenos empiristas", esto es, empiristas no fundacionistas. Cf. McDowell, J., Having the World in View.
} 


\section{La crítica de Dewey al empirismo tradicional y la concepción experimental de la experiencia}

En lo que sigue, quisiera presentar la que — según creo- podría considerarse como la crítica deweyana al Mito de lo Dado. Mi pretensión no es exegética; antes bien, pretendo reconstruir las críticas de Dewey al empirismo tradicional como una crítica alternativa al mentado Mito. Con ello aspiro (i) a resaltar algunas ventajas de la posición de Dewey y (ii) a mostrar la actualidad de su concepción de la experiencia para algunas discusiones recientes sobre teoría de la percepción ${ }^{12}$. Como lo adelanté en la Introducción, en esta sección reconstruiré la crítica de Dewey al empirismo tradicional a fin de caracterizar la concepción deweyana de la experiencia. Ello sentará las bases para especificar, en la sección siguiente, cómo puede entenderse este aspecto de la filosofía de Dewey como una crítica al Mito de lo Dado.

La filosofía de Dewey puede ser entendida como una nueva forma de empirismo $^{13}$. Su reconstrucción de la noción de experiencia toma en cuenta dos factores: el surgimiento de las ciencias experimentales, y el desarrollo de una psicología basada en la biología ${ }^{14}$. Aunque Dewey reconoce la importancia de la tradicional

${ }^{12}$ El presente artículo puede leerse como una contribución —en el específico contexto de la epistemología - a la reivindicación de la obra de Dewey que varios intérpretes están llevando a cabo actualmente. Para una valoración de conjunto de la obra de Dewey, Cf. por ejemplo Bernstein, R., Filosofía y democracia: John Dewey, Barcelona, Herder, 2010. Para el impacto que la obra de Dewey ha tenido en la filosofía del propio Bernstein, véase Del Castillo, R., «Derivas pragmatistas», que constituye la introducción del libro recién mentado. Sobre la noción de experiencia en el ámbito de la política y de la ética, Cf. Del Castillo, R., "John Dewey and the Ethics of Recognition», en Hickman, L., Flamm, M., y Skowroñski, K., (eds.), The Philosophy of John Dewey, Amsterdam-New York, Rodopi, 2010.

13 Bernstein, R., John Dewey, Atascadero, Ridgeview Letterpress \& Offset Inc., 1966, p. 45. Considero que el empirismo de Dewey es compatible (aunque no idéntico) con el «empirismo mínimo» de McDowell (Cf. McDowell, J., Mind and World, Cambridge, Harvard University Press, 1994). Si bien Dewey ha enfatizado siempre el papel de la acción en la experiencia, y este énfasis no aparece en Mind and World, ambas concepciones parecen tener en común esto: la pretensión de rehabilitar el carácter epistemológico de la experiencia sin incurrir en lo que Sellars llama «el Mito de lo Dado». Por lo demás, recientemente McDowell se ha acercado más a una posición deweyana al responder a las acusaciones de intelectualismo. Cf. McDowell, J., "What Myth?» y «Response to Dreyfus» ambos en McDowell, J., The Engaged Intellect, Cambridge, Harvard University Press, 2009.

${ }_{14}$ Dewey, J., La reconstrucción de la filosofía, Barcelona, Planeta-Agostini, 1986, p. 108. 
noción empirista de «experiencia» (esto es, como corte final de apelaciones para toda pretensión de conocimiento), sostiene, no obstante, que los empiristas han fracasado a la hora de desarrollar una concepción de la experiencia que esté en sintonía con el espíritu experimental de la ciencia. En particular, han fracasado en su intento por presentar una concepción de la experiencia que se ajuste a la experiencia, pues, según lo entiende Dewey, han introducido un divorcio insalvable entre la razón y la experiencia que no se condice con los hechos. Por el contrario, la atención a los métodos y resultados de las ciencias experimentales, junto con la nueva orientación biológica de la psicología, nos permite advertir — señala Deweyla íntima continuidad que existe entre la experiencia y la razón.

En «The Need for a Recovery of Philosophy», Dewey sintetiza el blanco de sus críticas. En la concepción tradicional, la experiencia se describe como:

a) Primariamente, un asunto de conocimiento;

b) Algo psíquico y subjetivo;

c) Atada exclusivamente al pasado, vinculada a lo que ha sido dado en el pasado;

d) Compuesta originariamente por entidades particulares (ideas simples), con lo cual se implica que las conexiones y continuidades provienen de una instancia ajena a la experiencia misma; y

e) Una instancia antitética del pensamiento, con lo cual se implica que toda inferencia — ajena en principio a la experiencia— involucra un salto más allá de esta ${ }^{15}$.

A estos rasgos que la tradición ha atribuido a la experiencia, Dewey opone aquellos que, según su concepción, son propios de la noción reconstruida de la experiencia.

a') Antes que una cuestión de conocimiento, «la experiencia es un asunto de intercambio de un ser vivo con su entorno físico y social»;

b’) La experiencia involucra desde el principio «un mundo objetivo que entra en juego en las acciones y sufrimientos de los hombres», y que "sufre modificaciones a través de sus respuestas»;

15 Dewey, J., «The Need for a Recovery of Philosophy», McDermott, J. (ed.), The Philosophy of John Dewey, Chicago, The University of Chicago Press, 1973, p. 61. 
c') La experiencia «en su forma vital es experimental, un esfuerzo por cambiar lo dado». Se caracteriza por «la proyección, por adelantarse hacia lo desconocido; su rasgo preeminente es la conexión con un futuro»;

d') La experiencia «que es estar expuesto al entorno, sufrir y luchar por controlarlo en nuevas direcciones», lejos de ser un conjunto de átomos recombinables desde el exterior (esto es, por la razón), «está preñada de conexiones»; y

e’) La experiencia «está repleta de inferencia. No hay, aparentemente, experiencia conciente sin inferencia» ${ }^{16}$.

Permítaseme considerar cada punto con algún detenimiento. Considerada desde el punto de vista de la biología, la experiencia es, primariamente, un modo de interactuar con el entorno. En tal sentido, está al servicio de la adaptación del organismo a su medio, del control de aquellas cosas y sucesos que son hostiles a la vida. De este punto de partida se desprenden casi todos los rasgos que Dewey atribuye a la experiencia: antes que al conocer, la experiencia sirve a la adaptación; puesto que el organismo que tiene experiencias está situado desde el principio en su entorno natural y social, la experiencia supone la existencia de un mundo objetivo; y, dado que está al servicio del control de las contingencias del entorno, la experiencia supone un esfuerzo por cambiar lo que le es dado al organismo y por anticipar los resultados de sus acciones.

Detengámonos en el punto a’). Para Dewey, la experiencia es primariamente un proceso por el cual somos afectados por el entorno como consecuencia de nuestras acciones. El organismo nunca es un receptor pasivo, un mero espectador, sino un agente que trata de cambiar el medio para alcanzar sus propósitos: «La experiencia significa primariamente no conocimiento, sino formas de hacer y sufrir. El conocer debe ser descrito descubriendo qué modo particular — cualitativamente único- de hacer y sufrir es» ${ }^{17}$. Lo que afecta al organismo no constituye

${ }^{16}$ Dewey, J., «The Need for a Recovery of Philosophy», p. 61.

17 Dewey, J., «The Need for a Recovery of Philosophy», p. 78. Y más adelante: «La cosa a ser conocida no se presenta primariamente como una materia de conocimiento-y-error en absoluto. Ocurre como un estímulo para la acción y como la fuente de ciertas afecciones. Es algo a lo cual reaccionar $(\ldots)$ y también algo que reacciona de maneras inesperadas a nuestras reacciones $(. .$. Tal presencia en la experiencia no tiene nada que ver con el conocimiento o la conciencia (...) aunque tiene todo que ver con el conocimiento y la conciencia en el sentido de que los últimos dependen de la experiencia previa de esta clase no cognitiva», pp. 84-85. Cf. también Dewey, J., La busca de la certeza, México, FCE, 1952, p. 192. 
una muestra ociosa e indiferente de información acerca de lo que sucede en el mundo; antes bien, es un estímulo para actuar de una manera determinada, es «un factor directivo en la adaptación de la vida al medio circundante» ${ }^{18}$. Como elemento conciente — señala Dewey— la sensación indica una interrupción en un curso de acciones previamente iniciadas, es una señal para volver a dirigir la acción. Considérese un ejemplo sencillo: mientras todo funciona debidamente, la persona que escribe no siente la presión del lápiz sobre el papel o sobre sus dedos. Su atención no se centra en ello, sino en la actividad que está ejecutando. Las sensaciones aquí funcionan como estímulos para el ajuste automático de la actividad. Si la punta del lápiz se rompe, se experimenta un choque consciente, se advierte que se ha interrumpido la actividad que se estaba desarrollando. Miramos el lápiz, lo afilamos y volvemos a la actividad que estábamos realizando. Como puede verse a través de este ejemplo, la experiencia conciente supone algún tipo de actividad en la que se insertan las sensaciones en tanto estímulos para el ajuste automático de la conducta. Cuando la actividad se ve interrumpida por algún cambio en el entorno, la atención se focaliza en ese cambio. La experiencia de la punta del lápiz tiene como propósito reestablecer la actividad. Por eso dice Dewey: «Las sensaciones no son partes de ningún conocimiento (...) Son provocaciones, estímulos, apremios para un acto de investigación que habrá de acabar en conocimiento» ${ }^{19}$. Tal como interpreto estas afirmaciones de Dewey, no es que, en el proceso de adaptación al medio, la experiencia no provea información alguna al organismo, o que no involucre el pensamiento. Si así fuera, no sería posible el ajuste flexible de su conducta al entorno. Antes bien, lo que pretende destacar Dewey en contra de la entera tradición moderna es que la vinculación originaria del organismo con su entorno no es teórica o cognoscitiva, sino práctica. Distorsionamos nuestra experiencia si la concebimos como exclusivamente dirigida al conocer. La experiencia está primariamente al servicio de la coordinación de la acción, de la adaptación. En consecuencia, cuando Dewey habla de experiencia «no cognitiva» o «irreflexiva», sólo está señalando que el interés primario de la experiencia no es el conocer ${ }^{20}$. Así pues, cuando la relación del organismo con su medio ambiente es fluida y su actividad en él se desarrolla sin obstáculos, la experiencia provee estímulos para el ajuste automático de la conducta. En este caso, el foco de la atención está concentrado en la actividad misma, en el objetivo a alcan-

${ }^{18}$ Dewey, J., La reconstrucción de la filosofía, Barcelona, Planeta-Agostini, 1986, p. 111.

19 Ibid., p. 113.

${ }^{20}$ Cf. Bernstein, R., John Dewey, p. 62. 
zar, no en lo que nos revela la experiencia. Se trata de lo que podríamos llamar «experiencia en la acción», pues en este tipo de casos, la experiencia sólo provee insumos para poder realizar la actividad que estamos haciendo ${ }^{21}$.

Pero además, naturalmente, la experiencia puede adquirir un valor cognoscitivo $^{22}$. Esto sucede cuando el objeto de la experiencia es considerado concientemente en cuanto tal. La consideración conciente de un estímulo que provee la experiencia no surge de la nada, sino de una situación que se ha vuelto indeterminada ${ }^{23}$. Ante tal indeterminación, el organismo no sabe cómo responder: «No sólo la respuesta es incierta, sino que el estímulo lo es igualmente; uno es incierto sólo en la medida en que lo es el otro» ${ }^{24}$. El pensamiento reflexivo se inicia ante el problema de descubrir cuál es el estímulo correcto y cuál ha de ser la respuesta adecuada a tal estímulo. Para volver a nuestro ejemplo: inesperadamente, dejamos de poder escribir. ¿Qué ha pasado? ¿Cómo reaccionar a ello? ¿Cómo restablecer

${ }^{21}$ En mi opinión, hay aquí espacio para concebir en Dewey esta forma primaria de experiencia no cognoscitiva, y las habilidades involucradas por ella, como conceptuales en un sentido parecido al enfatizado por Noë y McDowell. Cf. Noë, A., Action in Perception, Cambridge, The MIT Press, 2004, cap. 6, y McDowell, J., «What Myth?», en McDowell, J., The Engaged Intellect, Cambridge, Harvard University Press, 2009. En efecto, tal como interpreto este punto en la filosofía de Dewey, las sensaciones que tenemos cuando desarrollamos cualquier actividad, y los objetos percibidos, nos permiten ajustar nuestros movimientos precisamente porque poseen algún tipo de significado. Su significatividad proviene del hecho de que participan de un todo mayor que es la actividad que estamos llevando a cabo. Para volver a un ejemplo ya considerado: las sensaciones visuales y táctiles que tenemos mientras escribimos en un papel con un lápiz hacen posible, ciertamente, el ajuste de nuestros movimientos. Para poder realizar tales actividades, es preciso advertir, ciertamente, cuál es el uso del lápiz, del papel, y algunas de las propiedades de cada una de estas cosas (por ejemplo, que el lápiz puede ser tomado por la mano, que el papel es de tal naturaleza que puede escribirse sobre él, etc.).

${ }^{22}$ Sobre la distinción entre la experiencia como adaptación o ajuste y la experiencia como cognoscitiva, véase, por ejemplo, lo que dice aquí Dewey: «Cuando el agua es un estímulo adecuado para la acción o cuando sus reacciones nos oprimen y nos abruman, permanece fuera del alcance del conocimiento. Cuando, sin embargo, la presencia desnuda de la cosa (...) cesa de operar directamente como estímulo para la respuesta y comienza a operar en conexión con la previsión de las consecuencias que tendrán lugar cuando se responda a ellas, comienza a adquirir significado —a ser conocido, a ser un objeto- . Es notado como algo que es húmedo, fluido, que satisface la sed, disipa dificultades, etc (...) En la medida en que el estímulo visual opera como un estímulo solamente, no hay aprehensión, ni un notar en absoluto el color o la luz. A la mayor parte de los estímulos sensoriales reaccionamos precisamente de esta manera completamente no cognitiva». Dewey, J., «The Need...», p. 85.

23 "Como elemento conciente la sensación señala una interrupción en un curso de actividades anteriormente iniciadas», Dewey, J., La reconstrucción de la filosofía, p. 111-112.

${ }^{24}$ Dewey, J., «The Reflex Arc Concept in Psychology», p. 145. 
la actividad? Es en ese momento que prestamos atención a lo que vemos, y así notamos que la punta del lápiz se ha roto. La atención ha dejado de estar puesta en la actividad de escribir, y ha pasado a las cualidades de los objetos de la experiencia. En este contexto, puede decirse que la acción es puesta al servicio de la experiencia, pues la única forma de determinar el estímulo, según Dewey, consiste en tomar nota de lo que nos revelan las consecuencias de nuestras acciones referidas al objeto en cuestión. Se trata de lo que podría denominarse, en contraposición a la actitud anterior, «la acción en la experiencia», pues de lo que se trata, ahora, es de una actividad al servicio del conocimiento, del descubrimiento — por medio de la experiencia— de aquellas cualidades del medio que están impidiendo una relación determinada, fluida, con él.

No hay aprendizaje si no hay un actuar y un sufrir las consecuencias de ese actuar, y si no se advierten las correlaciones entre esas dos instancias. He aquí, por ejemplo, lo que dice Dewey:

«Las cualidades sensibles son importantes, pero cobran una significación intelectual únicamente como consecuencia de actos realizados deliberadamente [...] Las cualidades sensibles experimentadas por la vista poseen su condición y su misión cognoscitiva no (como sostienen los empiristas sensualistas) en y por sí mismas aisladamente, como algo que meramente se impone a la atención, sino porque son las consecuencias de operaciones definidas y deliberadamente practicadas. Sólo en conexión con el propósito o la idea de esas operaciones pueden significar algo, ya sea para descubrir un hecho o para contrastar una teoría ${ }^{25}$.

Es cuando actuamos en vistas de un fin cognoscitivo, para determinar ciertos hechos o probar alguna hipótesis, cuando modificamos el entorno para testear nuestras ideas, que la experiencia adquiere su completo significado cognoscitivo: nos revela qué consecuencias se siguen de nuestras intervenciones, cómo el mundo nos afecta o afectaría si actuamos o actuásemos de tal y cual manera guiados por nuestras ideas.

Consideremos el punto b'). El punto de partida de Dewey es el organismo en su entorno natural y social. Es allí en donde hay que examinar "empírica-

25 Dewey, J., La busca de la certeza, pp. 98-99. 
mente» la experiencia. La concepción tradicional, en cambio, comienza considerando «especulativamente» la experiencia como un tipo de estado mental perteneciente a un sujeto cognoscente que se halla fuera del mundo real ${ }^{26}$. En tales circunstancias, la experiencia es pensada como un estado interno y privado del sujeto, con un status epistémico privilegiado y en principio caracterizable independientemente del mundo externo. Esta concepción —elaborada y fomentada por lo que Dewey llama «la industria de la epistemología»— es la que genera los tradicionales problemas con los que han lidiado los filósofos desde la modernidad hasta la actualidad. Ahora bien, si la idea misma de la experiencia como algo que está "fuera» del mundo natural es un dogma de la filosofía especulativa, una idea que es contraria a los hechos, entonces las interminables discusiones acerca de cómo la mente puede conocer el mundo externo es un problema sin sentido. No merece, pues, una respuesta sino un liso y llano abandono ${ }^{27}$. Para Dewey, la doctrina de la continuidad biológica ha mostrado la irrelevancia empírica de la concepción empirista tradicional de la experiencia: «Si el desarrollo biológico es aceptado, el sujeto de la experiencia es al menos un animal, continuo con otras formas orgánicas en un proceso de organización más compleja ${ }^{28}$. Desde la perspectiva de Dewey, pues, el sujeto de experiencia es un organismo situado en el mundo. El contenido de la experiencia está dado por ese mismo mundo con el que interactúa. No hay un interior a la mente y un exterior a ella. No hay un problema del mundo externo. La experiencia no es subjetiva (al menos, en el sentido tradicional). Ni siquiera es idéntica a la actividad cerebral ${ }^{29}$. El sujeto de la experiencia es el organismo entero en sus interacciones con el entorno natural y social. Así pues, cuando consideramos que el sujeto de conocimiento está situado desde el principio en el mundo, y advertimos que primariamente se trata de un sujeto práctico, que ha de lidiar con ese entorno, el conocer se torna un modo derivado de interactuar con la realidad ${ }^{30}$.

${ }^{26}$ Dewey, J., «The Need for a Recovery of Philosophy», p. 71.

27 Ver Dewey, J., «The Influence of Darwinism on Philosophy», en McDermott, J., (ed.), op. cit., p. 41.

${ }^{28}$ Dewey, J., "The Need for a Recovery of Philosophy», p. 78.

29 Para versiones más actualizadas y sorprendentemente cercanas a la de Dewey, Cf. Gibson, J., The Ecological Approach to Visual Perception, New Jersey, Laurence Earlbaum Associates, Publishers, 1979, y Noë, A., Action in Perception, Cambridge, The MIT Press, 2004.

30 «Si es verdad que el yo o el sujeto de experiencia es parte y parcela del curso de eventos, se sigue que el yo deviene un conocedor. Deviene una mente en virtud de una forma distintiva de participar en el curso de los eventos. La distinción significativa no es más entre un conocedor y el mundo; es 
Veamos el punto c'). La experiencia, según Dewey, no es mera afección, recepción pasiva de estímulos. Frente a lo que denomina «el modelo del espectador» ${ }^{31}$, de acuerdo al cual el sujeto de la experiencia es un sujeto pasivo que contempla desinteresadamente el mundo, la experiencia es, para Dewey, «un asunto de hacer y sufrir simultáneos ${ }^{32}$. Puesto que el organismo ha de introducir cambios en el entorno para poder adaptarse, puesto que la experiencia es una manera de interactuar con el medio, y puesto que no hay control del entorno sin anticipación y expectativas, la experiencia está inescindiblemente conectada con la acción y, en consecuencia, con el futuro. «La anticipación es por tanto más primaria que el recuerdo; la proyección que la evocación del pasado; lo prospectivo que lo retrospectivo" ${ }^{33}$. Desde este punto de vista, conocer es considerar las posibilidades futuras que una situación existente presenta ${ }^{34}$.

Ya en «Reflex Arc Concept in Psychology», Dewey había mostrado la estrecha conexión entre acción y experiencia. En el análisis de la experiencia, no debemos pensar - aduce Dewey- el estímulo sensorial, la actividad central y la descarga motora como entidades separadas y completas en sí mismas. Antes bien, se trata de distinciones funcionales pertenecientes a un proceso más amplio y unitario que es la coordinación. Dice Dewey:

El análisis nos revela que empezamos, no con un estímulo sensorial, sino con una coordinación sensorio-motora, la coordinación óptico-ocular, y que en cierto sentido es el movimiento lo que es primario y la sensación secundaria, donde el movimiento de los músculos del cuerpo, la cabeza y ojos determina la cualidad de lo que se experimenta. (...) El verdadero comienzo está en el acto de ver; es un mirar, no una sensación de luz. El quale sensorial nos da el valor del acto $^{35}$.

Para Dewey, pues, el estímulo sensorial, en tanto posee algún valor, está siempre precedido por una coordinación sensorio-motora. Si algo se revela como

entre diferentes formas de ser en [el mundo] y de movimiento de las cosas; entre una forma física bruta y una propositiva, inteligente», Dewey, J., "The Need for a Recovery of Philosophy», p. 91.

31 Dewey, J., La busca de la certeza, México, FCE, 1952.

32 Dewey, J., "The Need for a Recovery of Philosophy», p. 63.

33 Ibid., p. 64.

34 Ibid., p. 91.

35 Dewey, J., "The Reflex Arc Concept in Psychology», pp. 137-138. 
caliente o capaz de quemarnos, es porque hemos acercado o podríamos acercar la mano. Si algo se revela como manipulable, es porque lo hemos tomado o podríamos tomarlo. Un sonido no es un mero estímulo; también es el acto de oír. En la recepción del sonido está implicado un determinado aparato motor que involucra el movimiento de la cabeza, la tensión de los músculos auriculares, etc. ${ }^{36}$.

En el mismo sentido, en La reconstrucción de la filosofía, dice Dewey:

El organismo no está (...) esperando que le salga algo. No espera, pasivo e inerte, a que algo exterior a él lo presione y moldee. El organismo actúa sobre las cosas que lo rodean (...) Los cambios que produce en ese medio circundante reaccionan a su vez sobre el organismo y sobre sus actividades. El ser viviente padece, sufre, las consecuencias de su propio obrar. Esta íntima conexión entre el obrar y el sufrir o padecer es lo que llamamos experiencia $^{37}$.

Esta es, precisamente, la que Dewey llama «concepción experimental de la experiencia ${ }^{38}$. De acuerdo a ella, no hay experiencia sin acción, pues es actuando sobre el medio que éste revela sus propiedades. Es advirtiendo las consecuencias de nuestro propio obrar que aprendemos algo del entorno.

Los puntos d') y e') destacan el carácter orgánico, holista de la experiencia. Contrariamente a la tradición del empirismo clásico, que sostiene que la experiencia está constituida por una colección de ideas simples o cualidades sensoriales que deben ser integradas por medio de leyes de asociación, la experiencia es - para Dewey - una totalidad orgánica articulada inferencialmente. La doctrina según la cual las ideas y sensaciones son existencias separadas no ha sido derivada —observa Dewey- de la observación o la experimentación ${ }^{39}$. En cuanto prestamos atención a lo que de hecho es la experiencia, se pone de manifiesto que la experiencia está, por sí misma, organizada. Este punto de vista hace innecesaria la intervención de una razón supra-empírica que, como en el sistema kantiano, sintetice la diversidad sensible. Para Dewey, «La experiencia encie-

36 Ibid., pp. 141-142.

37 Dewey, J., La reconstrucción de la filosofía, p. 110.

38 Ibid., p. 117.

39 Dewey, J., «The Need for a Recovery of Philosophy», p. 67. 
rra dentro de sí misma los principios de conexión y de organización $»^{40}$. En vez de estar constituida por átomos que deben ser relacionados, «Los verdaderos «materiales» de la experiencia consisten en maneras adaptables de acción, en hábitos, funciones activas, conexiones entre el obrar y el sobrellevar; es decir, coordinaciones de sensaciones para el obrar ${ }^{41}$. Es en virtud de esa coordinación sensorio-motora que la experiencia deviene «reguladora de sí misma»" ${ }^{42}$. La acción es estimulada y controlada por las sensaciones; y éstas, a su vez, adquieren su sentido en virtud de la acción o, más precisamente, de las consecuencias de la acción. Es justamente en este punto donde se pone de manifiesto el papel de la inferencia en la experiencia. En efecto, en la medida que un organismo es capaz de tomar una sensación o un hecho como signo de otra cosa, de algo que no está dado en el presente, de algo que se revelará a través de las consecuencias de su acción, es capaz de inferir ese hecho futuro a partir de lo que experimenta en el presente y, así, deviene capaz de controlar el futuro: «La extensión de la capacidad de un agente para inferir, del poder de usar un hecho dado como un signo de algo que no está dado, da la medida de la extensión de su habilidad sistemática para incrementar su control del futuro» ${ }^{43}$. Veamos, ahora, cuáles son las implicaciones que tiene esta teoría de la experiencia para el «Mito de lo Dado».

\section{Dewey y el Mito de lo Dado}

¿En qué sentido la concepción deweyana de la experiencia implica una crítica a lo que Sellars llama «el Mito de lo Dado»? Recordemos que el núcleo de dicho Mito está constituido por la idea según la cual episodios internos que no involucran conceptos (y que, por tanto, no pertenecen al espacio lógico de las razones) constituirían, no obstante, instancias de conocimiento y, en consecuencia, podrían ser utilizados para justificar estados conceptualmente articulados como las creencias.

Pues bien, si prestamos atención a la concepción de la experiencia de Dewey, podremos ver que la idea de algo meramente dado en la experiencia, que no pre-

${ }^{40}$ Dewey, J., La reconstrucción de la filosofía, p. 114.

${ }^{41}$ Ibid., p. 114.

${ }^{42}$ Ibid., p. 117.

43 Dewey, J., "The Need for a Recovery of Philosophy», p. 69. 
supone o guarda relación alguna con otras sensaciones, ideas o sentimientos, no puede constituir conocimiento alguno. Dicho en otros términos, no hay nada que, en la experiencia, cumpla las siguientes dos condiciones: a) que sea meramente dado y b) que constituya una instancia de conocimiento. Y, además, precisamente porque en la experiencia no hay inmediatez, no hay algo que sea simplemente dado, el contenido de la experiencia no puede ofrecer un fundamento último para el conocimiento empírico, tal como había pretendido el empirismo tradicional. En virtud de estos dos puntos — sostengo— la noción deweyana de experiencia involucra una crítica y un rechazo del «Mito de lo Dado».

En efecto, aunque Dewey rechaza la idea kantiana de una síntesis conceptual y a priori de la diversidad sensible, considera, no obstante, que las sensaciones son mudas si no están conectadas unas con otras; o si los objetos de la percepción no son vinculados con las posibles consecuencias a las que podrían dar lugar si interactuásemos con ellos ${ }^{44}$. En este sentido, puede sostenerse que existe un elemento kantiano en la teoría de la experiencia de Dewey, aunque naturalizado: la idea de que la experiencia es una totalidad organizada, interpretada, «sintetizada». Dicha "síntesis» (si es que cabe esta palabra aquí) no es llevada a cabo por una razón supra-empírica, como en Kant, sino por las acciones mismas del organismo que interactúa con su entorno. Obsérvese lo que dice Dewey al respecto:

La actividad constructiva u organizadora del «pensamiento» no es adquirida por éste como una función trascendental, una forma o modo del ego, la mente o la conciencia supra-empírica, sino que es adquirida por el pensamiento como una actividad vital ${ }^{45}$.

De modo semejante a Kant, aunque sin la postulación de un ámbito trascendental, Dewey reconoce, pues, que la experiencia supone el pensamiento ${ }^{46}$.

${ }^{44}$ La experiencia, al estar esencialmente ligada a la acción, involucra, además de simple afección, intencionalidad, propósito. Como dice Faerna, «Las sensaciones se tienen, pero las observaciones se hacen». Faerna, A., Introducción a la teoría pragmatista del conocimiento, Madrid, S. XXI, 1996. p. 206.

45 Dewey, J., «Experience and Objective Idealism», en Dewey, J., The Influence of Darwin on Philosophy and Other Essays, New York, Prometheus Books, 1965, p. 211.

46 «El hecho de que el pensamiento es un rasgo intrínseco de la experiencia es fatal para el empirismo tradicional que hace de él un producto artificial», Dewey, J., "The Need...», p. 70. 
Pero, en su caso, la organización que provee el pensamiento consiste en la vinculación de experiencias actuales con experiencias futuras que podrían tener lugar si actuáramos de cierta manera. Tenemos aquí dos tesis mutuamente implicadas: la de que la experiencia consiste, simultáneamente, en actuar y verse afectado como consecuencia de ese actuar ${ }^{47}$; y la de que el sentido de lo experimentado proviene de su vinculación con otros objetos que podrían ser experimentados también. Lo que hace posible esa vinculación es la acción del agente y la recepción de las consecuencias de su obrar. Hay, pues, una mutua implicación de estos dos elementos: sin la acción sería imposible advertir la significatividad de lo experimentado; pero, al mismo tiempo, sin la significatividad de lo experimentado, sin las vinculaciones que podrían establecerse entre lo que se experimenta en el presente y lo que podría experimentarse en el futuro, la acción sería ciega, carecería de lo que Dewey llama «inteligencia» ${ }^{48}$.

Respecto del carácter interpretativo de la acción, dice, por ejemplo, Dewey:

Es tan cierto que la respuesta motora determina el estímulo como que el estímulo sensorial determina el movimiento. De hecho, el movimiento es sólo con vistas a determinar el estímulo, a fijar de qué tipo de estímulo se trata, a interpretarlo [...] Cuál sea concretamente y en un momento dado la sensación dependerá, pues, enteramente de cómo se esté dirigiendo la actividad. No tiene una cualidad fija. La búsqueda del estímulo es la búsqueda de las condiciones exactas de la acción, esto es, del estado de cosas que decida cómo debe completarse una coordinación que comienza ${ }^{49}$.

Pero el actuar «abre» un plexo de significados, dota de sentido a lo experimentado, sólo en la medida en que se ponen en conexión el actuar y sus consecuencias. Son las correlaciones entre sensaciones y acciones lo que otorga a lo experimentado su significado. Dicho en otros términos, es advirtiendo qué tipo

47 Esta tesis, omnipresente en la obra de Dewey, está emparentada con la teoría ecológica de la percepción de Gibson y, más recientemente, con el llamado «enfoque enactivo» de la percepción de Noë y Hurley.

48 «El uso de lo dado o terminado para anticipar la consecuencia del proceso que está teniendo lugar es precisamente lo que es significado por «ideas», por «inteligencia». Dewey, J., «The Need...", p. 69.

49 Dewey, J., «The Reflex Arc Concept in Psychology», McDermott, J., (ed.), op. cit., pp. 141 y 146 . 
de consecuencias vienen asociadas a nuestras acciones que podemos otorgarle a lo experimentado un significado particular:

La experiencia (...) es primariamente lo que se sufre en conexión con actividades cuya importancia reside en sus consecuencias objetivas — su relación con experiencias futuras (...)—. Lo que está simplemente «ahí», es de interés únicamente en las potencialidades que podría indicar. Como terminado, como completamente dado, no explica nada. Pero como un signo de lo que podría venir, se convierte en un factor indispensable en la conducta que trata con los cambios, el resultado del cual no está todavía determinado ${ }^{50}$.

He aquí por qué la idea de algo meramente dado resulta completamente extraña a la noción de experiencia de Dewey. Cualquier objeto, suceso, sensación o propiedad experimentada posee un significado para el organismo sólo en la medida en que indica o es un signo de - para ese mismo organismo- otro objeto, suceso, sensación o propiedad que podría experimentarse en el futuro ${ }^{51}$. Elimínense esas posibles conexiones entre tales entidades, y ya no habrá para el organismo experiencia de algo qua algo determinado, significativo. Por eso dice Dewey:

Ser conciente [acquainted] de una cosa es estar seguro (desde el punto de vista de la experiencia misma) de que es de tal y tal carácter; que se comportará, si se da la oportunidad, de tal y cual manera; que el rasgo obvia y flagrantemente presente está asociado con otros rasgos que se mostrarán en sí mismos si se sigue la guía del rasgo presente. Ser conciente es anticipar en alguna medida sobre la base de la experiencia previa ${ }^{52}$.

\section{Y en otro lugar}

Una experiencia es un conocimiento, si en su quale hay una distinción y conexión experimentada de dos elementos de la siguiente clase: uno sig-

${ }^{50}$ Dewey, J., «The Need for a Recovery of Philosophy», pp. 68-69.

51 La tesis deweyana de que los objetos, propiedades y eventos, en tanto experimentados, son signos o significan otros objetos, propiedades o eventos para el sujeto, se encuentra esparcida por toda la obra de Dewey. Cf. «The Experimental Theory of Knowledge», pp. 83, 88, 90, 94, 99 y 107; «The Need for a Recovery of Philosophy», pp. 68, 69 y 84; La busca de la certeza, pp. 110, 187, 206 y 207.

52 Dewey, J., «The Experimental Theory of Knowledge», pp. 82-83. 
nifica o apunta a la otra de la misma manera en que ella misma está presente, mientras que la otra es esa que, mientras que no está presente de la misma manera, debe devenir así presente si el significado o la intención de su compañera ha de ser satisfecha a través de la operación establecida ${ }^{53}$.

Así pues, la sensación sola y aislada, que "está simplemente ahí», sin conexión alguna con otra cosa, carece de significado. Es, en el mejor de los casos, solamente un estímulo ${ }^{54}$. El conocimiento perceptivo involucra constitutivamente un conjunto de remisiones que conectan lo experimentado con lo que podría experimentarse si se actuara de un modo determinado 55 . De hecho, podrían especificarse los contenidos de la experiencia — en tanto experiencia reflexiva, cognoscitivarecurriendo a condicionales que conectaran, en sus antecedentes, las acciones $\mathrm{u}$ operaciones a realizar sobre el objeto en cuestión, y en sus consecuentes, los resultados de tales operaciones ${ }^{56}$. Por ejemplo, bajo la hipótesis de que lo que estoy viendo es una manzana, entonces puedo ensayar una serie de experimentos simples que involucran los condicionales antedichos: si muerdo la manzana, entonces tendré que experimentar determinado sabor; si presiono sobre ella con un cuchillo, deberá cortarse, etc. La experiencia experimental revela, así, no sólo cómo las cosas nos afectan o afectarán si realizamos determinadas acciones, sino también cómo interactúan las cosas entre sí (en nuestro caso, el cuchillo con la manzana $)^{57}$. Toda experiencia perceptiva, en la medida en que su contenido resulta

53 Ibid., p. 90.

54 Pero téngase en cuenta lo dicho en la nota 21.

55 "Cuando tratamos de cerciorarnos de la naturaleza de un objeto confuso y poco familiar, llevamos a cabo varios actos con el fin de establecer una nueva relación con ese objeto, de modo que salgan a relucir cualidades que nos ayuden a entenderlo", Dewey, J., La busca de la certeza, p. 75 .

56 «Las cualidades sensibles son importantes, pero cobran una significación intelectual únicamente como consecuencia de actos realizados deliberadamente [...] Las cualidades sensibles experimentadas por la vista poseen su condición y su misión cognoscitiva no (como sostienen los empiristas sensualistas) en y por sí mismas aisladamente, como algo que meramente se impone a la atención, sino porque son las consecuencias de operaciones definidas y deliberadamente practicadas», Dewey, J., La busca de la certeza, pp. 98-99. Una idea semejante puede hallarse en otro pragmatista, Lewis, C. I., Mind and the World Order, New York, Dover Publications, 1929, cap. V.

${ }^{57}$ Diversas objeciones pueden suscitarse en este punto. Por ejemplo, podría señalarse que los mentados condicionales no son constitutivos del contenido de la experiencia perceptiva, sino que sólo revelan una dependencia causal entre la experiencia y las acciones. Sin embargo, el punto de Dewey — tal como lo interpreto- es que no sabríamos con qué objeto estamos tratando en la experiencia si no conociéramos al menos algunos de tales condicionales. Quien no conoce ningu- 
significativo para el agente, supone un entramado más o menos extenso compuesto por condicionales que vinculan posibles acciones sobre el objeto con posibles consecuencias como su resultado. El contenido de la experiencia sobrepasa lo meramente dado, involucra ciertas expectativas acerca del posible comportamiento de los objetos que experimentamos, y esas expectativas tienen que ver, justamente, con las diversas formas en que tales objetos podrían afectarnos y podrían afectarse entre sí si lleváramos a cabo determinadas operaciones.

Esta suerte de «holismo» hace de telón de fondo, pues, de la crítica deweyana del atomismo perceptivo. En este punto, y aunque de distinto modo, Dewey y Sellars están de acuerdo. Por ejemplo, dice Sellars:

Uno no puede tener conocimiento observacional de ningún hecho a menos que sepa muchas otras cosas también [...] el conocimiento observacional de cualquier hecho particular, i.e. que esto es verde, presupone que uno conoce hechos generales de la forma $X$ es un sintoma confiable de $Y$. $Y$ admitir esto requiere un abandono de la idea empirista tradicional de que el conocimiento observacional «se sostiene sobre sus propios pies» ${ }^{58}$.

Por su parte, Dewey afirma:

No hay aprehensión sin algún (...) contexto; no hay conciencia que no sea reconocimiento o expectativa (...) La conciencia siempre implica un poco de familiaridad; un trazo de reconocimiento, de anticipación bienvenida o temida del rasgo que sigue ${ }^{59}$.

no de los condicionales involucrados en la experiencia de una manzana, no sabe lo que está percibiendo. Otra objeción posible podría señalar que la idea misma de un experimento supone la percepción de las condiciones requeridas para realizar dicho experimento (por más simple que sea) y que, por tanto, la acción no puede ser esencial al contenido de la experiencia. Frente a esto, quiero insistir en que, independientemente de todo movimiento, de toda acción exploratoria, no hay contenido significativo en la experiencia. Las manipulaciones de los objetos de su entorno que vemos realizar a los niños pre-lingüísticos no son manipulaciones de objetos cuyos significados ya comprenden por el sólo hecho de estar en frente de ellos. Tales manipulaciones revelan las cualidades de los objetos y, por tanto, el sentido de lo que experimentan. La manipulación de, por ejemplo, un plato revela ciertas propiedades de ese objeto: que se ve de distintas formas según la perspectiva que se adopte, que tiene un determinado peso, que es duro, etc.

${ }^{58}$ Sellars, W., Empiricism and the Philosophy of Mind, \# 36, pp. 75-76.

59 Dewey, J., «The Experimental theory of Knowledge», pp. 79-80. 
Con todo, lo que más distingue a las posiciones de Dewey y Sellars es el giro lingüístico. Para Sellars:

Toda conciencia de clases, parecidos, hechos, etc., dicho brevemente, toda conciencia de entidades abstractas — en verdad, aun toda conciencia de particulares - es un hecho lingüístico. De acuerdo a él [el nominalismo psicológico], ni siquiera la conciencia de tales clases, parecidos y hechos como perteneciendo a la así llamada experiencia inmediata está presupuesta por el proceso de adquirir el uso del lenguaje ${ }^{60}$.

Y en el parágrafo 16 de «Empiricism...» Sellars sugiere que las experiencias involucran aserciones o afirmaciones proposicionales (la experiencia de ver que algo es tal y cual incluye, además de ciertas sensaciones visuales, la proposición o aserción de que el algo es tal y cual ${ }^{61}$. Esto, sin duda, suscita el problema de cómo se relacionan las sensaciones con las aserciones de suerte que, como resultado, el sujeto vea, por ejemplo, que esto es un cubo de hielo rosa. Hasta donde puedo ver, este problema no queda resuelto por el sólo hecho de recurrir a una teoría adverbial de la percepción ${ }^{62}$. Pero, aun cuando este problema pudiera resolverse satisfactoriamente, quedaría por dilucidar cómo, a partir de la articulación de las sensaciones y las aserciones (o pensamientos) causados, ambos, por los objetos físicos, logramos tener experiencias de tales objetos ${ }^{63}$.

60 Sellars, W., Empiricism and the Philosophy of Mind, \# 29, p. 63.

${ }^{61}$ Sellars, W., Empiricism and the Philosophy of Mind, \# 16, pp. 39 y ss. En "The Structure of Knowledge», dice: "Ver que esto es un cubo de hielo rosa involucra un pensamiento de que esto es un cubo de hielo rosa», p. 303. Sin embargo, unas líneas más abajo Sellars aclara que el pensamiento ha de ser entendido en términos de oraciones en el lenguaje del «habla interna» o «mentalés», y que tales oraciones no deben ser confundidas con imágenes verbales. Estas expresiones sugieren que, aunque Sellars sigue pensando que la percepción involucra oraciones, estas no son oraciones pertenecientes al lenguaje ordinario. Cf. Neri Castañeda, H., (ed.), Action, Knowledge and Reality. Studies in Honor of Wilfrid Sellars, Indianápolis, The Bobbs-Merrill Company, Inc., 1975.

${ }^{62}$ Cf. Sellars, W., «The Structure of Knowledge», Neri Castañeda, H., op. cit.

${ }_{63}$ No puedo desarrollar esta observación aquí, pero el punto es el siguiente: si la experiencia es una combinación de sensaciones y proposiciones, como dice Sellars, entonces resulta difícil explicar cómo podemos ser concientes, a través e la experiencia, de los objetos físicos que, supuestamente, causan dichas sensaciones y proposiciones. La experiencia perceptiva quedaría encapsulada dentro de episodios internos constituidos por sensaciones y pensamientos. Esto suscita el clásico problema del mundo externo. 
En cambio, para Dewey, la experiencia nos provee una apertura al mundo ${ }^{64}$, es experiencia de los objetos físicos del entorno. En la experiencia no somos inmediatamente concientes de datos sensoriales o proposiciones, sino de los objetos y eventos del entorno en tanto que estos constituyen signos de otras cosas. El contenido de la experiencia está constituido, en parte, por los objetos mismos que percibimos, $y$, en parte, por las vinculaciones regulares que somos capaces de rastrear entre tales objetos y otras cosas. El contenido perceptivo se constituye, no necesariamente en virtud del lenguaje, sino, más en general, en virtud del estatus de signo que los objetos adquieren para el sujeto, esto es, en la medida en que el agente es capaz de anticipar experiencias futuras, de entrever las remisiones implicadas por lo experimentado. La experiencia no involucra una afirmación (aunque su contenido podría ser expresado por una afirmación); antes bien, contiene una sensación, objeto o evento que posee el estatus de un signo, esto es, que remite a otra cosa: un cierto aroma es reconocido como distintivo de cierta clase de comida; el color que adquiere cierto líquido en el laboratorio indica ciertas características de esa sustancia; la sensación de calor en la mano indica la presencia del fuego; el hecho de que haya huellas en la playa señala el paso, por ese lugar, de alguien. En todos estos casos, la experiencia perceptiva posee su significado en virtud de las vinculaciones sígnicas entre lo actualmente experimentado y lo que podría experimentarse en el futuro. Tales conexiones son las que hacen posible, para el sujeto de experiencia, inferir lo que sucederá o podría suceder a partir de lo experimentado en el presente. Esto resulta de crucial importancia tanto para lidiar con las cosas de una forma inteligente ${ }^{65}$, como a la hora de conocer el mundo ${ }^{66}$. En ambos casos, la inferencia sigue el rastro de las relaciones causales entre las cosas (incluido nuestro cuerpo) o, al menos, las regularidades observadas en el entorno.

${ }^{64}$ Smith, J., America's Philosophical Vision, Chicago, University of Chicago Press, 1992, p. 31.

65 «Un ser que puede usar hechos dados y terminados como signos de cosas por venir; que puede tomar cosas dadas como evidencias de cosas ausentes, puede, en esa medida, prever el futuro; puede formar razonablemente expectativas. Es capaz de forjar ideas; posee inteligencia. Pues el uso de lo dado o terminado para anticipar la consecuencia del proceso que está teniendo lugar es precisamente lo que es significado por "ideas", por "inteligencia”. Dewey, J., «The Need for a Recovery of Philosophy», p. 69.

66 «La inferencia, el uso de lo que sucede para anticipar lo que sucederá —o podría sucederhace la diferencia entre la participación dirigida y no dirigida. Y esta capacidad para inferir es precisamente la misma que la del uso de los acontecimientos naturales para el descubrimiento y determinación de las consecuencias (...) que constituyen el conocimiento», Dewey, J., «The Need for a Recovery of Philosophy», p. 70. 
Ahora bien, por más logrado que sea el equilibrio que pueda alcanzar el hombre con su entorno, por más bien que su experiencia se ajuste al mundo, dicho equilibrio siempre será precario, pues ese entorno — tanto natural como sociales inestable, está en continuo cambio, comporta peligros siempre nuevos ${ }^{67}$. De ahí que el ideal de una búsqueda filosófica de la certeza (que, para Dewey, es la causa de las dicotomías filosóficas tradicionales, tales como conocimiento vs acción, teoría $v$ s práctica, etc.) sea una mera ilusión. El falibilismo peirceano constituye uno de los pilares de la teoría de la experiencia y de la investigación de Dewey.

De un modo ciertamente diferente, otro ejemplo del holismo y anti-fundacionismo de Dewey puede hallarse en "Propositions, Warranted Assertibility, and Truth». Allí, en respuesta a ciertas objeciones presentadas por Russell a su concepción de la investigación ${ }^{68}$, Dewey cuestiona la tesis de que "hay proposiciones que son conocidas en virtud de su propia presencia directa inmediata ${ }^{69}$, como «Rojez-aquí». Frente a las pretensiones atomistas y fundacionistas de Russell, Dewey objeta: (i) que "aquí» posea un significado autónomo, completamente independiente del significado de "allí». La distinción entre «aquí» $\mathrm{y}$ «allí» entraña determinaciones que van más allá de cualquier cosa dada directamente; (ii) si, como parece sostener Russell, el carácter puro y último de las llamadas "proposiciones básicas» descansa en el hecho de que objetos del tipo «rojez-aquí» son concebidos como presencias sensibles directas, entonces — arguye Deweypareciera que la seguridad de la verdad de una proposición básica como «Rojezaquí» depende del hecho de que sepamos que las presencias sensibles directas que la fundamentan son efectivamente presencias sensibles. Pero esto - sigue el argumento- supone una teoría psicológica sumamente elaborada que explique a) cuáles son los procesos que conectan causalmente la proposición básica con la presencia sensible directa; y b) cuál es la conexión entre un sensum y el aparto sensorial corporal en virtud de la cual se determina que cierta cualidad dada es un sensum. Puesto que a) y b) suponen procesos inferenciales, la tesis de que exis-

${ }^{67}$ Cf. Dewey, J., Experience and Nature, London, George Allen \& Unwin, Ltd., 1929, cap. 2, y La busca de la certeza, caps. 1 y 2.

${ }_{68}$ Russell, B., Investigación sobre el significado y la verdad, Bs As, Losada, 2003.

69 Dewey, J., «Propositions, Warranted Assertibility, and Truth», Later Works of John Dewey, Jo Ann Boydston (ed.), Carbondale y Edwardsville, Southern Illinois University Press, 1991, vol. 14. Cito por la traducción de Faerna, A., en Faerna, A., (ed.), Dewey. La miseria de la epistemología. Ensayos de pragmatismo, Madrid, Biblioteca Nueva, 2000, p. 135. 
te algo así como «conocimiento directo» de ciertas cualidades sensoriales, y proposiciones básicas que constituyen la base de todo el edificio del conocimiento empírico, se torna insostenible.

En suma, desde el punto de vista de Dewey, lo meramente dado en la experiencia carece de valor, de significado, si no se encuentra vinculado con otros objetos, propiedades o eventos. Dicho en otros términos, aquello que es experimentado posee significado en la medida en que se constituye, para el sujeto de experiencia y acción, en un signo de otra cosa. La experiencia posee algún contenido, pues, en la medida en que lo experimentado indica o remite a otra cosa que podría ser experimentada; esto es, en la medida en que podemos inferir lo que sucederá a partir de lo que estamos experimentando ahora. Ser conciente —en la experiencia - de algo es advertir que ese algo se comportará de tal y cual manera si acontece la oportunidad, que el rasgo presente de algo está asociado con otros rasgos que se mostrarán en el futuro si se ejecutan determinadas acciones. Esto quiere decir que la fuente del significado no está primariamente en el lenguaje - como parece ser en Sellars - sino en la experiencia en tanto que en ella asociamos objetos y propiedades entre sí, en tanto que somos capaces de tomar un objeto como signo de otra cosa, o una cosa como signo de las consecuencias que provocará o podría provocar. $\mathrm{Y}$, dado que la experiencia siempre remite a otras experiencias, dado que está cargada de expectativas e inferencias, no hay nada en ella que garantice la certeza última del conocimiento.

Por lo expuesto hasta aquí considero que la concepción experimental de la experiencia de Dewey, interpretada como lo he hecho en este artículo, puede tener relevancia para ciertas discusiones actuales en filosofía de la mente y en epistemología; en particular, para aquellas que conciernen a la naturaleza y significado epistemológico de la experiencia perceptiva. Por ejemplo, el actual enfoque enactivo de la percepción recupera (no siempre recordando explícitamente la obra de Dewey) la tesis de que percibir es una forma de actuar, una manera de explorar el entorno. Además, los enfoques naturalistas de la experiencia perceptiva atienden indudablemente a la recomendación deweyana de estudiar experimentalmente la percepción y en estrecha vinculación con la biología. Por otro lado, los debates en epistemología acerca del valor epistémico de la experiencia tienen un antecedente original e interesante en la obra de Dewey. Cierta forma de realismo directo — que en el caso de Dewey permite sortear (antes que resolver) el problema escéptico- y el carácter semiótico que he atribuido a la noción 
deweyana de la experiencia constituyen —en mi opinión- no sólo anticipaciones de desarrollos posteriores en esta área de la filosofía, sino aportes sumamente atendibles en las discusiones de hoy en día. En efecto, la teoría experimental de la experiencia de Dewey puede leerse como una variante no tradicional de empirismo, un empirismo que, por un lado, vincula estrechamente — quizá pueda decirse: "constitutivamente»— experiencia y acción; y que, por otro, articula un enfoque de la experiencia que le permite defender el status epistémico de ésta sin recaer en el Mito de lo Dado. Prima facie, la ventaja de una teoría semejante reside - a mi juicio- en los siguientes puntos: a) al hablar de objetos, propiedades y sensaciones que, en tanto experimentados, se constituyen en signos para el agente, puede otorgarse a la experiencia valor epistémico sin necesidad de atribuir al contenido perceptivo carácter proposicional (como es en el caso de Sellars). Esta amplitud que podemos otorgarle a la concepción deweyana de la experiencia permite con mayor facilidad atribuir experiencias perceptivas a ciertas criaturas no humanas, una atribución que seguramente puede ser respaldada por la evidencia empírica. b) La concepción deweyana de la experiencia entronca fácilmente con el actual talante anti-cartesiano de algunos enfoques sobre la percepción en filosofía de la mente y en epistemología. c) Al enmarcar su concepción experimental de la experiencia dentro del darwinismo, Dewey no sólo re-sitúa el estudio de la experiencia (ahora debemos ver la experiencia humana en una línea de continuidad con la de otros seres biológicos), sino que alumbra dimensiones de la experiencia inadvertidas por los filósofos modernos; en particular, el hecho de que la experiencia es, primariamente, una forma de tratar con las cosas (antes que una forma de conocerlas), una forma que involucra un tipo de conocimiento práctico (know how) antes que un conocimiento proposicional $(\text { know that })^{70}$.

${ }^{70}$ La tesis de que nuestra relación original o primaria con las cosas es de naturaleza práctica es un tópico presente en distintos filósofos de diversas tradiciones. Cf., por ejemplo, Heidegger, M., Ser y tiempo, Bs As, FCE, 1980; Taylor, CH., «La superación de la epistemología», en Taylor, CH., Argumentos filosóficos, Barcelona, Paidós, 1997. La teoría de la acción comunicativa de Habermas ha sido considerada - especialmente por el propio Habermas - como una forma de descentralizar a la epistemología dentro de la escena de la filosofía. Cf. Habermas, J., Teoría de la acción comunicativa, Madrid, Taurus, 1987. 


\section{Conclusión}

He tratado de mostrar que la concepción experimental de la experiencia de Dewey puede interpretarse como una crítica al llamado "Mito de lo Dado» y como una manera de rehabilitar, para la epistemología, la noción de «experiencia». Su posición difiere de la de Sellars en, al menos, los siguientes puntos:

1) El sentido de lo percibido no requiere del lenguaje (al menos, no en principio). La conciencia del mundo alcanzada en virtud de la experiencia perceptiva supone, antes que el lenguaje, la comprensión de signos (naturales y culturales) que hacen posible la identificación de algo en cuanto que algo. Si por «proposición» se entiende el contenido o significado de una oración declarativa, entonces puede decirse que, para Dewey, el contenido de la experiencia no involucra, al menos primariamente, un contenido proposicional. En la experiencia somos concientes de los objetos del medio en el que nos movemos, y podemos serlo porque somos capaces de advertir las remisiones significativas de aquello que percibimos.

2) En el contexto de la investigación, la experiencia posee una clara connotación cognoscitiva. Aunque no toda experiencia es cognoscitiva, puede devenir tal en contextos reflexivos, en los que el agente está liberado de las urgencias de la acción. En cambio, no es en absoluto claro que en Sellars, especialmente en "Empiricism and the Philosophy of Mind», la experiencia sea per se cognitiva. En cualquier caso, aun cuando pudiera leerse a Sellars como diciendo que la experiencia es, en sí misma, una instancia de conocimiento (y no, como sostienen algunos intérpretes de su obra, la causa de una creencia), persistiría esta diferencia: para Dewey, la experiencia versa directamente acerca de los objetos de nuestro entorno. Sellars, en cambio, adhiere a una variante particular de la teoría adverbial de la percepción.

Si la tesis principal de este artículo — a saber, que la crítica de Dewey al empirismo clásico puede entenderse como una crítica al Mito de lo Dado- es correcta, entonces, no sólo contamos con una poderosa y actual línea de crítica al fundacionismo en la obra de uno de los principales representantes del pragmatismo, sino, también, con una teoría de la experiencia - la concepción experimental de la experiencia- que podría iluminar algunos de los debates recientes en epistemología acerca del carácter cognitivo de la percepción y de su significado para la justificación de la creencia. 


\section{Bibliografía}

Alston, W. (1983). «What's Wrong with Immediate Knowledge?», Synthese 55.

Bernstein, R. (1966). John Dewey, Atascadero, Ridgeview Letterpress \& Offset Inc.

- (2010). The Pragmatic Turn, Cambridge, Polity Press.

- (2010). Filosofía y democracia: John Dewey, Barcelona, Herder.

Bonevac, D. (2002). «Sellars vs the Given», Philosophy and Phenomenological Research, vol. LXIV, No 1.

Brandom, R. (1994). Making it Explicit, Cambridge, Harvard University Press.

- (1997). «Study Guide», en Sellars, W., Empiricism and the Philosophy of Mind, Cambridge, Harvard University Press.

Del Castillo, R. (2010). "Derivas pragmatistas», en Bernstein, R., Filosofía y democracia: John Dewey, Barcelona, Herder.

- (2010). "John Dewey and the Ethics of Recognition», en Hickman, L., Flamm, M. y Skowroñski, K. (eds.), The Philosophy of John Dewey, Amsterdam-New York, Rodopi.

DeWey, J. (1929). Experience and Nature, London, George Allen \& Unwin, Ltd.

- (1952). La busca de la certeza, México, FCE.

- (1965). «Experience and Objective Idealism», en Dewey, J., The Influence of Darwin on Philosophy and Other Essays, New York, Prometheus Books.

- (1965). "The Experimental Theory of Knowledge», en Dewey, J., The Influence of Darwin on Philosophy and Other Essays, New York, Prometheus Books.

- (1973). «The Need for a Recovery of Philosophy», McDermott, J. (ed.), The Philosophy of John Dewey, Chicago, The University of Chicago Press.

- (1973). "The Reflex Arc Concept in Psychology», McDermott, J. (ed.), op. cit.

- (1973). «The Influence of Darwinism on Philosophy», McDermott, J., (ed.), op. cit.

- (1986). La reconstrucción de la filosofía, Barcelona, Planeta-Agostini.

- (1991). «Propositions, Warranted Assertibility, and Truth», Later Works of John Dewey, Jo Ann Boydston (ed.), Carbondale y Edwardsville, Southern Illinois University Press, 1991, vol. 14. Cito por la traducción de Faerna, A., en Faerna, A., (ed.), (2000) Dewey. La miseria de la epistemología. Ensayos de pragmatismo, Madrid, Biblioteca Nueva. 
FAERNA, A. (1996). Introducción a la teoría pragmatista del conocimiento, Madrid, Siglo XXI.

Gibson, J. (1979.) The Ecological Approach to Visual Perception, New Jersey, Laurence Earlbaum Associates, Publishers.

Habermas, J. (1987). Teoría de la acción comunicativa, Madrid, Taurus.

Heidegger, M. (1980). Ser y tiempo, Bs As, FCE.

LEWIS, C. I. (1929). Mind and the World Order, New York, Dover Publications.

McDowell, J. (1994). Mind and World, Cambridge, Harvard University Press.

(2009). "What Myth?» en McDowell, J., The Engaged Intellect, Cambridge, Harvard University Press.

- (2009). «Response to Dreyfus» en McDowell, J., The Engaged Intellect, Cambridge, Harvard University Press.

- (2009). «Sellars on Perceptual Experience», en McDowell, J., Having the World in View, Cambridge, Harvard University Press.

- (2009). "Why Is Sellars's Essay Called "Empiricism and the Philosophy of Mind”?», en McDowell, J., Having the World in View, Cambridge, Harvard University Press.

Nö̈, A., (2004). Action in Perception, Cambridge, The MIT Press.

Rorty, R. (1979). Philosophy and the Mirror of Nature, Princeton, Princeton University Press.

- (1982). "Overcoming the Tradition: Heidegger and Dewey», en Rorty, R., Consequences of Pragmatism, Minneapolis, University of Minnesota Press.

- (1982). «Dewey’s Metaphysics», en Rorty, R., Consequences of Pragmatism, Minneapolis, University of Minnesota Press.

- (1998). «Dewey Between Hegel and Darwin», en Rorty, R., Truth and Progress, Cambridge, Cambridge University Press.

Russell, B. (2003). Investigación sobre el significado y la verdad, Bs As, Losada.

Sellars, W. (1997). Empiricism and the Philosophy of Mind, Cambridge, Harvard University Press.

- (1975) "The Structure of Knowledge», en Neri Castañeda, H., (ed.), Action, Knowledge and Reality. Studies in Honor of Wilfrid Sellars, Indianápolis, The Bobbs-Merrill Company, Inc. 
Smith, J. (1992). America's Philosophical Vision, Chicago, University of Chicago Press.

TAYLOR, CH. (1997). «La superación de la epistemología», en Taylor, CH., Argumentos filosóficos, Barcelona, Paidós.

Recibido: 9/05/2010

Aceptado: 14/10/2010 